\title{
Sistema de compostaje para el tratamiento de residuos de hoja de coca con la incorporación de tres activadores biológicos, en el centro experimental de Kallutaca \\ Composting system for waste treatment coca leaf with the addition of three biological activators in the Experimental Center Kallutaca
}

Apaza-Condori Emma Eva*, Mamani-Pati Francisco, Sainz-Mendoza Humberto

\begin{tabular}{|c|c|}
\hline Datos del Artículo & Resumen \\
\hline $\begin{array}{l}\text { Universidad Pública de El Alto-UPEA, } \\
\text { Área de Ciencias Agrícolas, Pecuarias y } \\
\text { Recursos Naturales, Ingeniería Agronómi- } \\
\text { ca. Laja-Los Andes - La Paz, Bolivia. } \\
\text { (591)2-2115231 } \\
\text { Instituto de Investigación y Extensión } \\
\text { Agrí́olla (IINEA). Area de Ciencias } \\
\text { Agrícolas, Pecuarias y Recursos Natura- } \\
\text { les, Ingeniería Agronómica } \\
\text { *Dirección de contacto: } \\
\text { Emma Eva Apaza-Condori. } \\
\text { Área de Ciencias Agrícolas, Pecuarias y } \\
\text { Recursos Naturales, Ingeniería Agronómi- } \\
\text { ca. La Paz, Bolivia. (591)2-2115231 } \\
\text { E-mail: evapazacondorie@gmail.com }\end{array}$ & $\begin{array}{l}\text { El objetivo del presente trabajo fue evaluar el proceso de compostaje de residuos de hoja de coca con la incorporación } \\
\text { de tres activadores biológicos (Yogurt, Suero de leche y Levadura). El presente trabajo se llevó acabo Centro Experi- } \\
\text { mental de Kallutaca, módulo de Bioabonos de la Carrera Ingeniería Agronómica de la Universidad Pública de El Alto, } \\
\text { Municipio de Laja La Paz. Los tratamientos planteados fueron: T1 (Hoja de coca + Yogurt); T2 (Hoja de coca + suero } \\
\text { de leche); T3 (Hoja de coca + levadura) y T4 (Testigo). El diseño utilizado fue completamente al azar con } 4 \text { tratamien- } \\
\text { tos y } 3 \text { repeticiones. } \\
\text { Los valores obtenidos en N se clasifican en niveles medios y altos, las cantidades de P, K se clasifican en niveles me- } \\
\text { dios. El valor obtenido } 7.9 \text { de pH, CE con } 12950 \mu \mathrm{S} / \mathrm{cm} \text { y la materia orgánica del } 61 \% \text { pertenecen al tratamiento T1. El } \\
\text { tiempo de descomposición fue de un período de } 105 \text { días que corresponde al tratamiento T3. }\end{array}$ \\
\hline \multirow[t]{3}{*}{$\begin{array}{l}\text { Palabras clave: } \\
\text { Compost, } \\
\text { Activadores biológicos, } \\
\text { hoja de coca. }\end{array}$} & \multirow[b]{2}{*}{ (C) 2015. Journal of the Selva Andina Biosphere. Bolivia. Todos los derechos reservados. } \\
\hline & \\
\hline & Abstract \\
\hline $\begin{array}{l}\text { J Selva Andina Biosph. } \\
\text { 2015; 3(2):75-85. }\end{array}$ & \multirow{3}{*}{$\begin{array}{l}\text { The objective of this study was to evaluate the composting process waste coca leaf with the addition of three biological } \\
\text { activators (yogurt, whey and yeast). This work was carried out Kallutaca Experimental Center, Biofertilizers module } \\
\text { Career Agricultural Engineering at the Public University of El Alto, La Paz municipality of Laja. Posed treatments } \\
\text { were: T1 (+ Yogurt Coca wastes); T2 (Coca wastes + whey); T3 (Coca wastes + yeast) and T4 (Control). The design } \\
\text { was completely randomized with } 4 \text { treatments and } 3 \text { repetitions. } \\
\text { The values in } \mathrm{N} \text { are classified medium and high levels the quantities of P, K are classified as middle levels. The value } \\
\text { obtained } 7.9 \mathrm{pH} \text {, EC } 12950 \mu \mathrm{S} / \mathrm{cm} \text { and } 61 \% \text { organic matter belong to treatment T1. The decomposition time was a } \\
\text { period of } 105 \text { days corresponds to treatment T3. }\end{array}$} \\
\hline $\begin{array}{l}\text { Historial del artículo } \\
\text { Recibido abril, 2015. } \\
\text { Devuelto noviembre, } 2015 \\
\text { Aceptado noviembre, } 2015 \text {. } \\
\text { Disponible en línea, noviembre } 2015 .\end{array}$ & \\
\hline $\begin{array}{l}\text { Editado por: } \\
\text { Selva Andina } \\
\text { Research Society }\end{array}$ & \\
\hline
\end{tabular}




\section{Introducción}

La producción de hoja de coca ha avanzado de forma consecutiva, llevando a cabo una creciente producción en los distintos departamentos de Bolivia (La Paz, Cochabamba y Santa Cruz), siendo la producción permitida por la Ley 1008, de 12000 ha Rodríguez (2011). Pero en los últimos estudios realizados por UNODC (2013), señala que hay aproximadamente 25300 ha plantadas de hoja de coca, lo cual significa que 13300 ha son cultivadas ilegalmente o bien destinados al narcotráfico UNODC (2013), es por ello que se procede a su incautación y su incineración.

Frente a esta situación, una de las alternativas de tratamientos de residuos orgánicos es el compostaje, que consiste en un proceso biooxidativo en la que intervienen poblaciones microbianas (bacterias, hongos y actinomicetos) en función a los factores que afectan el proceso (humedad, temperatura, $\mathrm{pH}$ ) y producto de ello se obtiene el compost, considerado un abono orgánico que contiene nutrientes que son asimilables para las plantas, garantizando rendimientos altos en los cultivos, mejora las propiedades físicas, químicas y biológicas del suelo (Soliva 2001).

Otro de los aspectos a tomarse en cuenta en el proceso de compostaje, es el tiempo de descomposición, para tal efecto Chilón (2010), utilizó activadores biológicos (yogurt, suero de leche y levadura), con la finalidad de disminuir el tiempo de compostaje de 7 a 3.5 meses.

El objetivo del presente trabajo fue evaluar el proceso de compostaje de residuos de hoja de coca con la 76 incorporación de tres activadores biológicos (Yogurt, Suero de leche y Levadura).

\section{Materiales y métodos}

El presente trabajo de investigación se llevó a cabo en la Estación Experimental Kallutaca de la carrera Ingeniería Agronómica - UPEA; ubicado en el municipio de Laja, provincia Los Andes. Geográficamente se sitúa entre $16^{\circ} 31^{\prime} 10^{\prime}$ ' latitud sur y $68^{\circ} 19^{\prime}$ 03', longitud oeste, a una altitud de $3901 \mathrm{msnm}$ forma parte del altiplano norte (Guarachi 2011).

Activadores locales (biológicos): Yogurt, suero de leche y levadura.

Hoja de coca residual. En el marco del convenio realizado con la Carrera Ingeniería Agronómica de la Universidad Pública de El Alto y el Viceministerio de Coca y Desarrollo Integral, Dirección General de la Hoja de Coca e Industrialización (DIGCOIN), se acopio la hoja de coca residual trasladado desde Villa Fátima, localizado específicamente en la calle Unduavi de la ciudad de La Paz. En el lugar se pesaba los bultos o taques cada uno constaba de $10 \mathrm{~kg}$, siendo registrada por la misma dirección, asimismo la hoja de coca procedía de los diferentes departamentos y provincias, tendiendo a variar en sus diferentes tamaños, color y el estado de la hoja de coca. La cantidad utilizada fue de 163 bultos o taques.

Estiércol de vaca e ingesta. A través de una coordinación efectuada con el matadero municipal de la ciudad de El Alto se pudo adquirir la ingesta para su uso, asimismo este producto se mezcló con el estiér- 
col de vaca, siendo adquirido de la Carrera de medicina Veterinaria y Zootecnia de la UPEA.

Calculo de los materiales

El método utilizado para la instalación fue el sistema Volumen de los residuos de hoja de coca. se utilizó "Indore. La formación de las 12 pilas para el proceso de compostaje estaban a $2.00 \mathrm{~m}$ de pila a pila. Cada pila tenía una dimensión de $2 \mathrm{~m}$ de ancho x 2 como referencia una lata de manteca que tiene la forma de un prisma recto. Coronel (2012), lo formuló de la siguiente manera:

$\mathrm{m}$ de largo x $1 \mathrm{~m}$ de alto.

$$
\begin{gathered}
\text { Volumen del prisma recto }=(\mathbf{a} * \mathbf{b} * \mathbf{c})=\mathrm{m}^{3} \\
V_{\text {residuosdehojade coca }}=(0.20 \mathrm{~m} * 0.20 \mathrm{~m} * 0.35 \mathrm{~m})=0.014 \mathrm{~m}^{3}
\end{gathered}
$$

La masa o la cantidad de los residuos de hoja de coca se pesó en la lata de manteca sobre una balanza analítica, obteniendo distintas cantidades y promediando se obtuvo $0.8 \mathrm{~kg}$.

Densidad de la hoja de coca residual, en función a la masa y el volumen hallado. Coronel (2012), lo expresa como:

$$
\begin{gathered}
\delta=\frac{m}{V}=\frac{\mathrm{kg}}{\mathrm{m}^{3}} \\
\delta_{\text {residuosde hojadecoca }}=\frac{0.8 \mathrm{~kg}}{0.014 \mathrm{~m}^{3}}=57.1 \mathrm{~kg} / \mathrm{m}^{3}
\end{gathered}
$$

El volumen estuvo en función al largo, ancho y altura de la pila, para la primera capa de residuos de hoja de coca:

$$
\begin{gathered}
\text { Volumen }=(\mathrm{a} * \mathrm{~b} * \mathrm{c})=\mathrm{m}^{3} \\
V_{\text {residuosde hojadecoca }}=2.00 \mathrm{~m} * 2.00 \mathrm{~m} * 0.30 \mathrm{~m}=1.2 \mathrm{~m}^{3} / \text { capa }
\end{gathered}
$$

Masa (cantidad), está en función a la densidad y el volumen. Coronel (2012), lo expresa como:

$\mathrm{m}=\delta * V$

$$
m_{\text {residuos de hojas decoca }}=57.1 \frac{\mathrm{kg}}{\mathrm{m}^{3}} * 1.2 \frac{\mathrm{m}^{3}}{\text { capa }}=68.5 \cong 68 \mathrm{~kg} / \mathrm{capa}
$$

Por pila se armó dos capas de un total de 136 kilos y para las 12 pilas fue un total de 1632 kilos de hojas de coca. Volumen del estiércol de vaca e ingesta (contenido ruminal), se utilizó como referencia una lata de manteca que tiene la forma de un prisma recto. Coronel (2012), lo formuló de la siguiente manera:

$$
\begin{gathered}
\text { Volumen del prisma recto }=(\mathrm{a} * \mathrm{~b} * \mathrm{c})=\mathrm{m}^{3} \\
\text { Volumen de la lata }=(0.20 \mathrm{~m} * 0.20 \mathrm{~m} * 0.35 \mathrm{~m})=0.014 \mathrm{~m}^{3}
\end{gathered}
$$


Densidad del estiércol de vaca e ingesta, en función a la masa y el volumen hallado. Coronel (2012), lo expresa como:

$$
\begin{gathered}
\delta=\frac{m}{V}=\frac{\mathrm{kg}}{\mathrm{m}^{3}} \\
\delta_{\text {estiercol de vaca e ingesta }}=\frac{10.00 \mathrm{~kg}}{0.014 \mathrm{~m}^{3}}=714.0 \mathrm{~kg} / \mathrm{m}^{3}
\end{gathered}
$$

La cantidad del estiércol de vaca (50\%) más la ingesta (50\%) se pesó en una balanza analítica, obteniendo distintas cantidades y promediando se obtuvo $10.00 \mathrm{~kg}$

El volumen estuvo en función al largo, ancho y altura de la pila, para la primera capa de estiércol de vaca e ingesta:

$$
V=2.00 \mathrm{~m} * 2.00 \mathrm{~m} * 0.20 \mathrm{~m}=0.8 \mathrm{~m}^{3} / \text { capa }
$$

Masa (cantidad), en función a la densidad y el volumen:

$$
\begin{gathered}
m_{\text {estiercolde vacae ingesta }}=714.0 \frac{\mathrm{kg}}{\mathrm{m}^{3}} * 0.8 \frac{\mathrm{m}^{3}}{\text { capa }}=571.2 \cong 571 \mathrm{~kg} / \mathrm{capa} \\
m_{\text {estiercoldevacasingesta }}=571 * 2=1142.4 \cong 1142 \mathrm{~kg} / \mathrm{pila}
\end{gathered}
$$

Por pila se armó dos capas de un total de $1142 \mathrm{~kg}$ y para las 12 pilas fue un total de $13704 \mathrm{~kg}$ de la mezcla de estiércol de vaca (50\%) e ingesta (50\%).

La cantidad está en función al porcentaje de humedad donde el estiércol de vaca es de un $86 \%$ y la ingesta de un $87 \%$, estudios realizados por Parra \& Castro (2004).

Activadores biológicos: Yogurt (Industrias PIL), Levadura Fleshmnan (Industrias Venado) y el Suero de leche procede de una vaca Holstein (Carrera de medicina Veterinaria y Zootecnia de la UPEA). Chilón (2010), indica que los derivados de la leche principalmente el yogurt y el suero de leche, así como la levadura, presentan un excelente efecto en la activación biológica del compost, disminuyéndose paulatinamente el tiempo de obtención del abono orgánico de 7 a 3.5 meses. Los activadores biológicos fueron utilizados directamente de sus envases sin realizar ningún tratamiento previo o análisis biológico, asimismo el agua utilizado fue potable para la preparación.

Preparación: Se mezcló 2 L de yogurt en 4 L de agua potable, $2 \mathrm{~L}$ de suero de leche en $4 \mathrm{~L}$ de agua $\mathrm{y}$ $500 \mathrm{~g}$ de levadura en $4 \mathrm{~L}$ de agua, siendo estas los tratamientos y como testigo solo se utilizó agua del lugar (Kallutaca).

Procedimiento

Se impermeabilizo toda la superficie del suelo con un agrofilm de $250 \mu \mathrm{m}$, para evitar la contaminación antrópica dentro de la pila de compost y de esa manera evitar ciertas impurezas que no están ligadas al producto final 
No se realizó ningún tratamiento previo a los residuos de hoja de coca. Las pilas de compostaje estuvieron compuestas de las siguientes capas: Residuos de hoja de coca humedecida con $2 \mathrm{~L}$ agua con $30 \mathrm{~cm}$ de altura, estiércol de vaca e ingesta con $20 \mathrm{~cm}$ de altura, residuos de hoja de coca humedecida con 2 litros agua con $30 \mathrm{~cm}$ de altura y por ultimo estiércol de vaca e ingesta con $20 \mathrm{~cm}$ de altura. Se utilizó para cada pila un callapo de $1.50 \mathrm{~m}$ para sostener la pila de compost, asimismo se pudo observar el desprendimiento de metano que asciende a la atmósfera.

Se usó el activador biológico seleccionado para cada pila y utilizando una regadera a razón de un litro se aplicó de forma uniforme sobre los residuos de hoja de coca en la primera y tercera capa de la pila de compostaje.

Finalizando se cubrió con el agrofilm de $250 \mu \mathrm{m}$ con una dimensión de $4 \mathrm{~m} \mathrm{x} 4 \mathrm{~m}$ a todas las pilas de compostaje, tendiendo a mantener la temperatura y evitar la evaporación. Este proceso no se lo realiza para la producción extensiva, por ser costoso el material.

Manejo en el proceso de compostaje:

Control de temperatura. La temperatura se midió cada siete días con ayuda de un geotermómetro o termómetro de suelo expresado en ${ }^{\circ} \mathrm{F}$, convirtiéndosela en ${ }^{\circ} \mathrm{C}$ desde una profundidad de $30 \mathrm{~cm}$ iniciando desde la parte superior, hasta considerar las partes laterales de la pila de compost, para ello se consideró horarios de mañana y tarde a las 08:30 am y a las 13:00 pm, efectuándose muestreos en cada unidad experimental para luego obtener promedios.

Aireación del sustrato o volteo. Los volteos de las pilas de compost se realizaron cada quince días, utilizando una pala, una picota y un rastrillo, para que el material que está en el núcleo del compost 79 quede afuera y el material que está afuera ingrese al interior o núcleo, estructurando de una forma uniforme los materiales teniendo así una descomposición adecuada en el proceso de compostaje.

Análisis de laboratorio. Para el análisis químico, se pesó un kilo de acuerdo a las normas que exige el laboratorio y se llevaron a analizar las doce pilas de compost a los Laboratorio de Calidad Ambiental (LCA - UMSA) y Servicios Analíticos - Laboratorios Químicos (Spectrolab-UTO).

Análisis estadístico. Ochoa (2009), para el cálculo del análisis de varianza se utilizó el paquete estadístico SAS (Statystical Análisis System) Ochoa (2009), el nivel de significancia que se utilizo fue del $5 \%$ y para las comparaciones entre tratamientos se utilizó la prueba de Duncan.

\section{Resultados}

\section{Fig. 1 Evolución de la temperatura registrada en el compost de Coca}

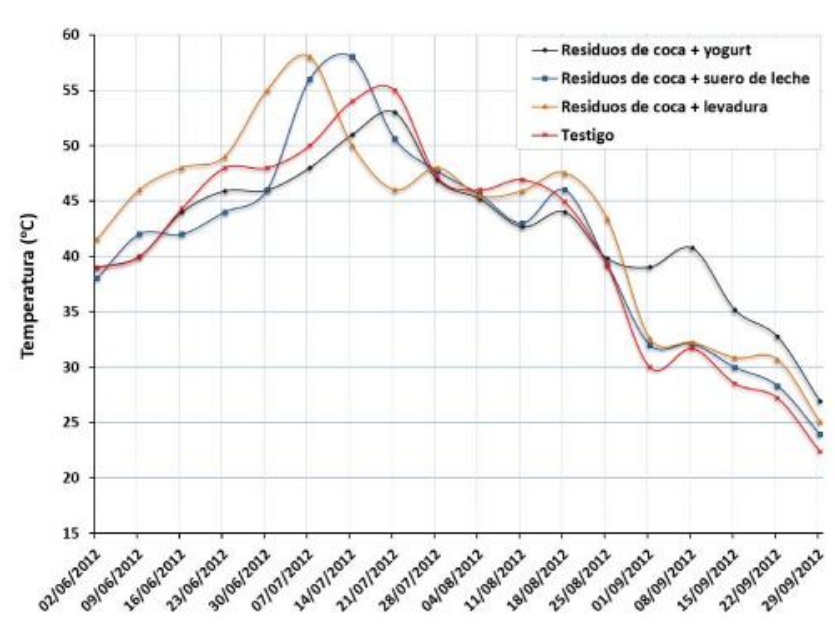

Tiempo de descomposición 
Tabla 1 Análisis físico-químico de la hoja de Coca

\begin{tabular}{lcc}
\hline & Hoja de Coca & \\
\hline Parámetros & Unidad & Valores \\
Nitrógeno total & $\%$ & 2.1 \\
Fósforo total & $\mathrm{mg} / \mathrm{kg}$ & 2744 \\
Sodio total & $\mathrm{mg} / \mathrm{kg}$ & 92 \\
Potasio total & $\mathrm{mg} / \mathrm{kg}$ & 12240 \\
Calcio total & $\mathrm{mg} / \mathrm{kg}$ & 9574 \\
Magnesio total & $\mathrm{mg} / \mathrm{kg}$ & 2548 \\
Materia orgánica & $\%$ & 94 \\
Cenizas & $\%$ & 6.0 \\
Proteína total & $\%$ & 13 \\
pH & & 4.9 \\
C.E. & & 4540 \\
\hline
\end{tabular}

Tabla 2 Análisis físico - químico del estiércol e ingesta de vaca

\begin{tabular}{lcc}
\hline & Estiércol e Ingesta & \\
\hline Parámetros & Unidad & Valores \\
Nitrógeno total & $\%$ & 1.6 \\
Fósforo total & $\mathrm{mg} / \mathrm{kg}$ & 9730 \\
Sodio total & $\mathrm{mg} / \mathrm{kg}$ & 7329 \\
Potasio total & $\mathrm{mg} / \mathrm{kg}$ & 19076 \\
Calcio total & $\mathrm{mg} / \mathrm{kg}$ & 9980 \\
Magnesio total & $\mathrm{mg} / \mathrm{kg}$ & 3200 \\
Materia orgánica & $\%$ & 77 \\
Cenizas & $\%$ & 23 \\
Proteína total & $\%$ & 10 \\
pH & $\mu .9$ & 4470 \\
C.E. & & \\
\hline
\end{tabular}

Tabla 3 Evaluación de las propiedades físico-químicos del compost

\begin{tabular}{|c|c|c|c|c|c|c|c|c|c|c|}
\hline \multicolumn{11}{|c|}{ Elementos Y Nutrientes Presentes En El Compost } \\
\hline & pH & C.E & M.O & $\begin{array}{c}\mathrm{N} . \\
\text { total }\end{array}$ & $\begin{array}{c}P . \\
\text { total }\end{array}$ & $\begin{array}{c}K . \\
\text { total }\end{array}$ & $\begin{array}{l}\text { Ca. } \\
\text { total }\end{array}$ & $\begin{array}{l}\text { Mg. } \\
\text { total }\end{array}$ & $\begin{array}{l}\text { Na. } \\
\text { total }\end{array}$ & $\begin{array}{c}\mathrm{Fe} . \\
\text { total }\end{array}$ \\
\hline Compost & & $(\mu \mathrm{S} / \mathrm{cm})$ & $(\%)$ & $(\%)$ & $\mathrm{mg} / \mathrm{kg}$ & $\mathbf{m g} / \mathbf{k g}$ & $\mathrm{mg} / \mathrm{kg}$ & $\mathrm{mg} / \mathrm{kg}$ & $\mathbf{m g} / \mathbf{k g}$ & $\mathrm{mg} / \mathrm{kg}$ \\
\hline T1 (Hoja de coca + Yogurt) & 7.9 & 12950 & 61 & 3.10 & 7886 & 19217 & 20017 & 5190 & 9715 & 9715 \\
\hline T2 (Hoja de coca + Suero de leche) & 7.8 & 12023 & 58 & 2.43 & 7058 & 18406 & 18816 & 5047 & 9034 & 9034 \\
\hline T3 (Hoja de coca + Levadura) & 7.8 & 11487 & 57 & 2.40 & 6839 & 17820 & 18087 & 5014 & 6536 & 6536 \\
\hline T4 (Hoja de coca +Agua) & 7.7 & 11020 & 56 & 2.33 & 5968 & 17671 & 17633 & 4772 & 5955 & 5955 \\
\hline
\end{tabular}




\section{Discusión}

Moreno \& Moral (2008) establecen que la temperatura es uno de los factores que influye de forma más crítica sobre la velocidad de descomposición de la materia orgánica durante el compostaje.

El comportamiento de la temperatura, donde el T3 (Residuos de coca + levadura), presentó una elevada temperatura de $42{ }^{\circ} \mathrm{C}$, ocasionando una transición de mesófila a termófila presentando una temperatura de $58{ }^{\circ} \mathrm{C}$ y por último en la tercera fase (fase de enfriamiento) se obtuvo $25^{\circ} \mathrm{C}$. Alcolea (2000), menciona que las variaciones de temperaturas dependen del tipo de materia orgánica que se utiliza. Los microorganismos que resulten beneficiados a una temperatura concreta son los que principalmente descompondrán la materia orgánica, produciéndose un desprendimiento de calor. Este calor provoca una variación de la temperatura en la pila, dependiendo del tamaño de la pila, de las condiciones ambientales y del volteo (Ekinci et al. 2004).

La etapa de estabilización comenzó a los 40 días aproximadamente para todas las pilas. Pero en el T2 (Residuos de coca + suero de leche) puede observarse que aumentó la temperatura inmediatamente y volvió a descender. Donde Silva et al. (En prensa), señalan que no se realizó una descomposición considerable de la materia orgánica, la cual contenía material que no fuera rápidamente degradado y a su vez permitiera el desarrollo de las bacterias termófilas.

La hoja de coca presenta los siguientes elementos y nutrientes: nitrógeno total $2.1 \%$; fósforo total $2744 \mathrm{mg} / \mathrm{kg}$, sodio total $92 \mathrm{mg} / \mathrm{kg}$, potasio total $12240 \mathrm{mg} / \mathrm{kg}$, calcio total $9574 \mathrm{mg} / \mathrm{kg}$, magnesio total $2548 \mathrm{mg} / \mathrm{kg}$, materia orgánica $94 \%$, ceniza 81
$6.0 \%$, proteína total $13 \%, \mathrm{pH} 4.9$ y conductividad eléctrica $4540 \mu \mathrm{S} / \mathrm{cm}$. Collazos et al. (1965), demostraron inicialmente la riqueza en minerales y vitaminas de la hoja de coca con "valores particularmente llamativos para el nitrógeno y el calcio" siendo esto los valores: nitrógeno $2.88 \%$, fósforo $405.0 \mathrm{mg}$, calcio $2196 \mathrm{mg}$ y ceniza $5.35 \%$.

Burton \& Turner (2003), consideran que la acumulación de estiércol en áreas determinadas de ganadería intensiva genera problemas como la emisión de malos olores, lixiviación a aguas subterráneas y superficiales, contaminación del suelo por aplicación de dosis excesivas, e incluso riesgos de enfermedades para los animales y para la salud humana.

El estiércol y la ingesta de vaca presenta los siguientes elementos y nutrientes: Nitrógeno total $1.6 \%$, fósforo total $9730 \mathrm{mg} / \mathrm{kg}$, sodio total 7329 $\mathrm{mg} / \mathrm{kg}$, potasio total $19076 \mathrm{mg} / \mathrm{kg}$, calcio total $9980 \mathrm{mg} / \mathrm{kg}$, magnesio total $3200 \mathrm{mg} / \mathrm{kg}$, materia orgánica $77 \%$, ceniza $23 \%$, proteína total $10 \%, \mathrm{pH}$ 8.9 y conductividad eléctrica $4470 \mu \mathrm{S} / \mathrm{cm}$. La FAO (2001), los estiércoles difieren mucho en su composición, dependiendo ésta de factores tales como clase, edad y características de cada animal, producción de leche (del ganado vacuno), cantidad y digestibilidad del forraje y alimentos concentrados consumidos por el ganado (el estiércol de los animales que se alimentan de forraje celulósico es más pobre en nutrientes para las plantas que el de aquellos que reciben raciones de alta concentración), la cantidad y tipo de las camas, duración y forma de almacenamiento y método de manejo. Moreno \& Moral (2008), el pH tiene una influencia directa en el compostaje debido a su acción sobre la dinámica de los procesos microbianos. 
Los tratamientos presentan un $\mathrm{pH}$ básico, donde se estima entre los 7.9-7.7. Benito et al. (2005), considera que el $\mathrm{pH}$ a medida que el proceso avanza, el valor va aumentando gradualmente hasta valores constantes que oscilaran entre 6.5 y 8.5 , dependiendo del material. Moreno \& Moral (2008), el aumento del pH es debido a la degradación de compuestos de naturaleza ácida y a la mineralización de compuestos nitrogenados hasta formar amoníaco, actuando también el proceso de amonificación como un importante sumidero de protones.

Los tratamientos presentan una alta conductividad eléctrica. La conductividad eléctrica de un compost está determinada fundamentalmente por su concentración de sales y en menor grado por la presencia de iones amonio o nitrato formados durante el proceso (Benito et al. 2005).

La CE tiende generalmente a aumentar durante el proceso de compostaje debido a la mineralización de la materia orgánica, hecho que produce un aumento de la concentración de nutrientes (SánchezMonedero et al. 2001).

Kiehl (1985), el conocimiento del contenido de los compost en materia orgánica es fundamental, pues se considera como el principal factor para determinar su calidad agronómica. Moreno \& Moral (2008), algunos compuestos procedentes de la materia orgánica son utilizados por los microorganismos para formar sus tejidos y otros son transformados en anhídrido carbónico y agua.

Los tratamientos presentan un porcentaje de materia orgánica, que está entre $61 \%-56 \%$. La materia orgánica descendiendo debido a su mineralización y a la consiguiente pérdida de carbono en forma de anhídrido carbónico; estas pérdidas pueden llegar a representar casi el $20 \%$ en peso de la masa com- postada (Zucconi \& De Bertoldi 1987). La velocidad de transformación de materia orgánica depende de su naturaleza física y química, de los microorganismos que intervienen y de las condiciones físico-químicas del proceso (humedad, aireación, temperatura y pH) (Michel et al. 2004).

El nitrógeno es un elemento esencial para la reproducción celular debido a la naturaleza proteica del protoplasma; se ha demostrado que la calidad de un compost como abono orgánico está directamente relacionada con su contenido de nitrógeno (Díaz et al. 2002).

De acuerdo a la clasificación por Costas et al. (1991), la cantidad de nitrógeno total en los tratamientos son considerados de un nivel alto y medio. Moreno \& Moral (2008), Los aumentos están relacionados con un efecto de concentración de amonio debido a la mineralización de la materia orgánica. Sánchez-Monedero et al. (2001), establecen que las transformaciones químicas que sufre el nitrógeno durante el compostaje vienen dadas por reacciones de amonificación, nitrificación y desnitrificación. Los microorganismos sólo pueden aprovechar compuestos simples, por lo que las moléculas más complejas se rompen en otras más sencillas (por ejemplo las proteínas en aminoácidos y estos en amoníaco) para poder ser asimiladas (Castaldi et al. 2005).

Moreno \& Moral (2008), El fósforo desempeña un papel fundamental en la formación de compuestos celulares ricos en energía, siendo necesario para el metabolismo microbiano. La cantidad de fósforo presente en los tratamientos están entre 7886 $\mathrm{mg} / \mathrm{kg}$ - $5968 \mathrm{mg} / \mathrm{kg}$. Se comprueba que, en general, entre el inicio y el final de la incubación se produce un aumento de las concentraciones de los distintos nutrientes, debido a la pérdida de materia 
orgánica de la masa a compostar (Díaz et al. 2004; Michel et al. 2004). Chilón (2010), alega que la mineralización del fósforo es análogo a la del nitrógeno, los compuestos polimerizados iníciales son desdoblados en compuestos simples en proteínas y aminoácidos hasta liberación de forma de ácido fosfórico, esto por microorganismos como hongos, bacterias y otros.

Moreno \& Moral (2008), los microorganismos implicados en el compostaje necesitan que una serie de nutrientes específico se encuentren en una forma química disponible, y a concentraciones adecuadas. Moreno (2005), el potasio es esencial a nivel metabólico y durante el proceso de división celular. De acuerdo a la clasificación por Costas et al. (1991), la cantidad de potasio total en los tratamientos son considerados de un nivel medio. Paco et al. (2004), mencionan que a medida que los microorganismos van descomponiendo los compuestos simples de la materia orgánica el potasio disminuye paulatinamente; al concluir el proceso de descomposición, los microorganismos mueren y los nutrientes aprovechados son mineralizados como óxido de potasio $\left(\mathrm{K}_{2} \mathrm{O}\right)$ para liberar el potasio alimentando en el compost con mayor carga de potasio al final.

\section{Conflictos de intereses}

La presente investigación fue financiada por la Universidad Pública de El Alto, Dirección de Investigaciones Ciencia y Tecnología (DICyT), siendo el intermediario el Instituto de Investigaciones y Extensión Agrícola (IINEA) de la Carrera Ingeniería Agronómica y no presenta conflictos de interés.

\section{Agradecimientos}

Los autores agradecen a la Universidad Pública de El Alto, Carrera Ingeniería Agronómica por habernos brindado los predios del Centro Experimental Kallutaca, para la ejecución del presente trabajo.

Al Instituto de Investigaciones y Extensión Agrícola (IINEA), por la colaboración y apoyo del presente trabajo a través del Ing. Laoreano Coronel Quispe.

A la Dirección de Investigaciones Ciencia y Tecnología (DICyT), por haberme dado el sustento económico para la ejecución del presente trabajo.

Al Viceministerio de Coca y Desarrollo Integral (VCDI), juntamente con la Dirección General de la Hoja de Coca e Industrialización (DIGCOIN) y la Unidad de Industrialización de la Hoja de Coca, por la colaboración, apoyo, confianza, por la oportunidad de trabajar y contribuir a la sociedad.

Al laboratorio de Calidad Ambiental-UMSA y a SPECTROLAB-UTO, por su colaboración en los análisis de muestras.

\section{Literatura citada}

Alcolea M. Manual de compostaje Domestico. Barcelona-España. 2004. Consultado 3 may. 2012. Disponible en www.compostando.com.

Benito M, Masaguer A, Moliner A, Cogger CG, Bary AI. Comparison of a gas detection tubes test with the traditional alkaline trap method to evaluate compost stability. Biol Fertil Soils. 2005; 41: 447-450. 
Burton CH, Turner C. Manure Management: Treatment Strategies for Sustainable Agriculture. Silso e Research Institute. Bedford (UK). 2003.

Castaldi P, Alberti G, Merella R, Melis P. Study of the organic matter evolution during municipal solid waste composting aimend at identifying suitable parameters for the evaluation of compost maturity. Waste Manag. 2005; 25: 209-213.

Chilón E. Compostaje altoandino, alimento al suelo vivo y cambio climático. CienciAgro. 2010; 2: 221-227.

Collazos CHC. Nutrición y coqueo. Rev Viernes Med. 1965; 16:(8)1. Lima. Consultado 24 feb. 2014. Disponible en http://www.congreso .gob.pe/congresista/2001/lguerrero/documentos /P.LHojadeCoca130904.pdf.

Coronel RL. Química General. La Paz, Bolivia. 2012; 22-479 pp.

Costa F, García C, Hernández T, Polo A. Residuos orgánicos urbanos. Manejo y utilización. Centro de Edafología y Biología Aplicada de la Segura. Consejo Superior de Investigaciones Científicas. Murcia. España. 1991; 88 pp.

Díaz MJ, Jiménez 1, Cabrera F, De Bertoldi M. Using a second order polynomials model to determine the optimum vinasse/grape marc ratio for in vessel composting. Compost Sci. Util. 2004; 12(3): 273-279.

Díaz MJ, Madejón E, Lopez F, López R, Cabrera F. Composting of vinasse and cotto gin waste by using two different systems. Resour Consery Recycl. 2002; 34(4): 235-248.

Ekinci K, Keener HM, Elwell DL. Effects of aeration strategies on the composting proccess: Part
I. Experimental studies. Trans. ASAE. 2004b; 47(5): 1697-1708.

FAO. (Organización de la Naciones Unidas para la Agricultura y la Alimentación). Manejo del suelo: producción y uso del compost en ambientes tropicales y subtropicales. Organización de las Naciones Unidas para la agricultura y la Alimentación. Roma. 2001; 177 pp.

Guarachi E. Balance hídrico en el cultivo de papa bajo condiciones de drenaje mixto en Suka Kollos en el centro experimental Kallutaca. Tesis Licenciatura. Universidad Pública de El Alto. La Paz, Bolivia. 2011; 20 pp.

Kiehl FJ. Fertilizantes orgánicos. Editora Agronómica Ceres Ltda. Sao Paulo. Brazil. 1985; $492 \mathrm{pp}$.

Michel FC, Jr Pecchia JA, Rigot J, Keener HM. Mass and nutrient losses during the composting of dairy manure amended with sawdust and Straw. Compost Sci Util. 2004; 12(14): 323334.

Moreno CJ, Moral HR. Compostaje. (Edit. Cient.). Ediciones Mundi-Prensa. Madrid-España. 2008; 238-320 pp.

Moreno J. Compostaje. En: Marín, I., Sanz, J. L., y Amils, R. (Eds.). Biotecnología y Medio ambiente. Ephemera, Madrid. 2005.

Ochoa TRR. Diseños Experimentales. 1ed. La Paz, Bolivia. 2009; 51-71 pp.

Paco G, Loza-Murguía M, Mamani F, Sainz H. Efecto de la Lombriz Roja Californiana (Eisenia foetida) durante el composteo y vermicomposteo en predios de la Estación Experimental de la Unidad Académica Campesina Carmen Pampa. J Selva Andina Res Soc. 2011; 2: 1439. 
Parra R, Castro D. Producción aeróbica de compost a partir de residuos de mataderos de reses y mercados. 2004. Consultado 20 mar. 2014. Disponible en http://www.cpts.org/proy investi/PROYECTO08,pdf.

Rodríguez A. Consejo Nacional de Lucha Contra el Tráfico Ilícito de Drogas - CONALTID. Programa de Apoyo al Control Social de la Producción de la Hoja de Coca Viceministerio de Defensa Social y Sustancias Controladas. Artes Gráficas Sagitario. 2011; 82 pp.

Sánchez-Monedero MA, Roig A, Paredes C, Bernal MP. Nitrogen transformation during organic waste composting by the Rutgers system and its effects on $\mathrm{pH}, \mathrm{EC}$ and maturity of the composting mixtures. Biores Technol. 2001; 78(3): 301-308.

Silva VJ, López MP, Valencia AP. Recuperación de nutrientes en fase sólida a través del compostaje. En prensa. Consultado el 7 de may. 2014. Disponible en http://www.bvsde.paho. org/bvsars/fulltext/compostaje.pdf.
Soliva M. Compostatge i gestió de residusorganics. Estudis i Monografies, 21. Servei de MediAmbient de la Diputació de Barcelona. 2001; $111 \mathrm{pp}$.

UNODC. Oficina de la Naciones Unidas la Droga y el Delito. 2013. Consultado 15 ago. 2012. Disponible enhttp://www.unodc.org/documents /cropmonitoring/Bolivia/BOLIVIA_Coca_Surv ey_2013_web.pdf.

Zucconi F, De Bertoldi M. Compost specifications for the production and characterization of compost from municipal solid waste. In Compost: Production, Quality and Use. ed. De Bertoldi, M., Ferranti, M.P., L'Hermite, P. and Zucconi, F. 1987; 30 pp. Elsevier Applied Science Publishers Ltd. 